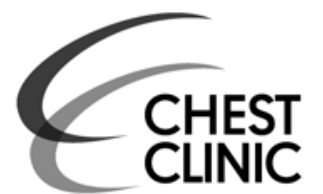

\section{Correspondence to}

Professor Jonathan Grigg, Blizard Institute, Barts and the London School of Medicine and Dentistry, Queen Mary, University of London, 4 Newark Street, London E1 2AT, UK; j.grigg@qmul.ac.uk

Received 12 June 2012 Accepted 22 June 2012 Published Online First 16 July 2012

\title{
The platelet activating factor receptor: a new anti-infective target in respiratory disease?
}

\author{
Jonathan Grigg
}

\begin{abstract}
There is increasing evidence that vulnerability to pneumococcal pneumonia is mediated by the expression of adhesion receptors for bacteria on lower airway cells. A key bacterial adhesion receptor is the plateletactivating factor receptor (PAFR). In vitro and animal studies have shown that upregulation of PAFR increases vulnerability to infection and blocking PAFR and knockdown of PAFR attenuates infection. Blocking PAFR may therefore be a novel therapeutic strategy in acute and chronic airway infection.
\end{abstract}

The lower airway contains a wide array of defenses against bacterial infection. In the larger airway, antibacterial defenses include mucociliary clearance, blood-derived mucosal cells and substances such as secretory $\operatorname{IgA}$, lysozymes, lactoferrins and defensins. In the alveoli, IgG, surfactant, alveolar macrophages and lymphocytes prevent inhaled bacteria gaining a foothold. The airway epithelium also presents a major physical barrier to bacterial disease. One way that bacteria evade this formidable barrier is to adhere to epithelial receptors. Strong adhesion to host-expressed receptors allows bacteria to be shuttled across the cell membrane as receptors are internalised.

Over the last decade, there is increasing evidence that the platelet-activating factor receptor (PAFR) is a major epithelial receptor used by respiratory bacteria to inveigle their way across the airway epithelium. During the 1990s PAFR, a G-protein coupled 7-transmembrane receptor for PAF, received significant attention as a potential target for asthma therapy. Orally active PAFR antagonists were tested in adults with asthma, but were then shelved. The first evidence for a role of PAFR in bacterial adhesion was provided by Cundell and colleagues ${ }^{1}$ who discovered that PAFR mediates the adhesion of virulent (but not non-virulent) Streptococcus pneumoniae to human airway epithelial cells in vitro. Pneumococcal adhesion to airway cells in Cundell's study was enhanced by interleukin (IL)$1 \alpha$ and blocked by a PAFR antagonist, whereas lowlevel basal pneumococcal adhesion to unstimulated airway cells was unaffected by a PAFR blocker. ${ }^{1}$ This pattern is compatible with the concept that adhesion of pneumococci to healthy airway cells is a relatively benign process leading, at most, to colonisation. In contrast, activation of cells results in upregulation of adhesion receptors that tend to facilitate bacterial invasion. For the pneumococcus, this latter pathogenic process involves an interaction between phosphorycholine (ChoP; a molecular mimic of PAF) in its cell wall binding to protein moieties in the PAFR on host cells. Some strains of non-typeable Haemophilus infleunzae, Pseudomonas aeruginosa and Acinetobacter baumannii also express ChoP-and therefore have the capacity to adhere to human bronchial epithelial cells in a PAFR-dependent manner. In vitro studies of human lower airway epithelial cells have found that rhinovirus, acid, fossil-fuel derived particulate matter, and most recently, cigarette smoke ${ }^{2}$ increase PAFR expression and enhance PAFR-dependent pneumococcal adhesion and invasion; that is, upregulation of PAFR expression on airway epithelium appears to be a non-specific response to a range of inhaled noxious stimuli.

If PAFR is a vulnerability factor for invasive pneumococcal disease, then animals with increased PAFR expression should be more vulnerable to severe pneumonia, and mice lacking the PAF receptor should be relatively resistant. Indeed, the PAFR knockout $\left(\mathrm{PAFR}^{-1-}\right.$ ) mouse is resistant to pneumococcal pneumonia with reduced mortality, less pneumococci in the lung, and less translocation of bacteria from the lung into the blood compared with the wild-type animal. Cundell and colleagues ${ }^{1}$ upregulated epithelial PAFR in vivo by instilling IL-1 into the airway of rabbits, and found that cytokine treatment increased vulnerability to pneumococcal lung infection. Recent data from a mouse model suggest that pneumococci per se via upregulation of the transcription factor $\mathrm{C} / \mathrm{EBP} \delta$, stimulate PAFR on airway cells - that is, pneumococci induce their own adhesion receptor. ${ }^{3}$ Once adherent pneumococci breach the epithelial barrier and enter the blood, they continue to co-opt PAFR to invade other organs. In contrast, no binding of the cell wall and no movement of the cell wall into other organs is found in the $\mathrm{PAFR}^{-/-}$ mouse. $^{4}$

To date, the role of anti-PAFR therapy in nontypeable $H$ infleunzae lung infection has not been assessed in an animal model, but ChoP-postive $P$ aeruginosa pneumonia infection in mice is attenuated by blocking PAFR. ${ }^{5}$ Although dysfunctional antibody production and defective splenic clearance is thought to underlie the vulnerability of patients with sickle cell disease (SCD) to invasive pneumococcal infection, a recent study suggests a role for PAFR in SCD. In the SCD mouse, constitutive PAFR expression by airway cells is increased, ${ }^{6}$ treatment with simvastatin reduces airway PAFR, and statin therapy reduces lung bacterial burden after pneumococcal infection. ${ }^{6}$ Similarly in the 


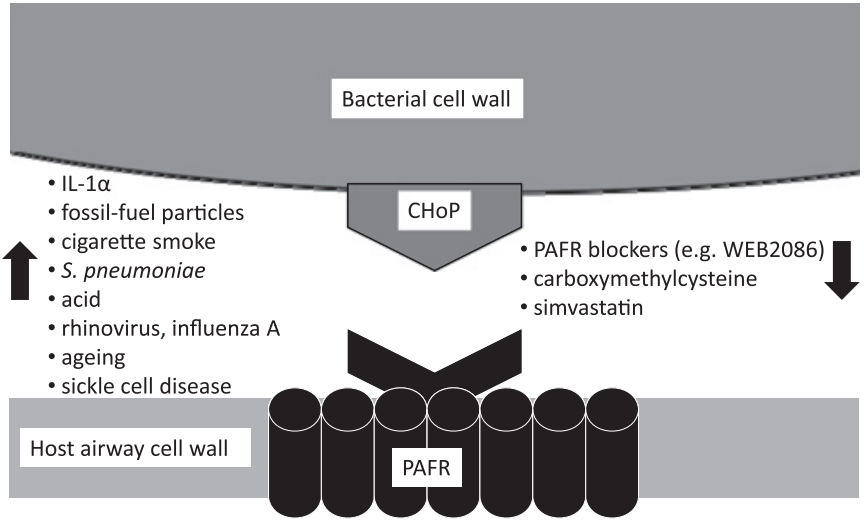

Figure 1 Putative factors influencing the adhesion of phosphorylcholine (ChoP) positive bacteria such as Streptococcus pneumoniae to lower airway cells via the platelet activating factor receptor (PAFR). IL, interleukin.

mouse model of vulnerability pneumococcal pneumonia in older people, ageing is associated with increased constitutive PAFR expression by airway cells-and an increased vulnerability to pneumococcal pneumonia. ${ }^{7}$ Viral infections predispose people to exacerbations of chronic infective conditions such as chronic obstructive pulmonary disease (COPD). The importance of PAFR in secondary pneumonia has been demonstrated in the mouse in which blocking PAFR reduces lethality from secondary pneumococcal pneumonia after influenza A. However, the beneficial effect of blocking PAFR in secondary bacterial infection after viral infection may be mediated by more than one mechanism, since activation of PAF (via PAFR) is a major driver of lethality from influenza, cerebral malaria and Dengue in animal models.

To date, very few studies have assessed airway PAFR expression in humans. Shirasaki and colleagues, ${ }^{8}$ reported that PAFR mRNA from heart-lung transplant donors is increased in people with asthma compared with normal controls. Shivshankar and colleagues ${ }^{7}$ reported an age-dependent increase in PAFR protein in lung biopsies from adults, and recently, we reported that bronchial epithelial PAFR protein expression is increased in healthy smokers compared with never-smokers. ${ }^{2}$ Conditions for which ChoP-expressing bacteria are present in the lower respiratory tract, for example COPD, are putative targets for PAFR therapy, but PAFR expression in patients with COPD is unknown. Indirect evidence for a role of PAFR in COPD is provided by a study of carboxymethylcysteine, a mucolytic that reduces COPD exacerbations. In human airway cells, pneumococcal adhesion is downregulated in vitro by carboxymethylcysteine via attenuation of PAFR. ${ }^{9}$

In conclusion, there is convincing evidence from in vitro studies and animal models that PAFR expression increases vulnerability to acute respiratory infection caused by some ChoP-expressing bacteria (summarised in figure 1). PAFR blockers are already available for use in humans, but before assessing their efficacy in therapeutic trials, more data on airway PAFR in health and disease are needed. Future research questions include: Does the expression of bacterial adhesion receptors by airway epithelial cells in COPD lie between a pattern supporting benign colonisation, and a pattern facilitating invasive disease? Do tightly adherent bacteria exist in the lower airway of conditions such as COPD-and is this a major source of free airway bacteria ?

\section{Competing interests None.}

Provenance and peer review Commissioned; internally peer reviewed.

\section{REFERENCES}

1. Cundell DR, Gerard NP, Gerard C, et al. Streptococcus pneumoniae anchor to activated human cells by the receptor for platelet-activating factor. Nature 1995;377:435-8.

2. Grigg J, Walters $\mathrm{H}$, Sohal SS, et al. Cigarette smoke and platelet-activating factor receptor dependent adhesion of Streptococcus pneumoniae to lower airway cells. Thorax. Published Online First: 1 May 2012. doi:10.1136/thoraxjnl-2011-200835

3. Duitman J, Schouten M, Groot AP, et al. CCAAT/enhancer-binding protein delta facilitates bacterial dissemination during pneumococcal pneumonia in a plateletactivating factor receptor-dependent manner. Proc Natl Acad Sci U S A 2012;109:9113-18.

4. Fillon S, Soulis K, Rajasekaran S, et al. Platelet-activating factor receptor and innate immunity: uptake of gram-positive bacterial cell wall into host cells and cell-specific pathophysiology. J Immunol 2006;177:6182-91.

5. Barbier M, Oliver A, Rao J, et al. Novel phosphorylcholine-containing protein of Pseudomonas aeruginosa chronic infection isolates interacts with airway epithelial cells. J Infect Dis 2008;197:465-73.

6. Rosch JW, Boyd AR, Hinojosa E, et al. Statins protect against fulminant pneumococcal infection and cytolysin toxicity in a mouse model of sickle cell disease. J Clin Invest 2010;120:627-35.

7. Shivshankar P, Boyd AR, Le Saux CJ, et al. Cellular senescence increases expression of bacterial ligands in the lungs and is positively correlated with increased susceptibility to pneumococcal pneumonia. Aging Cell 2011; 10:798-806.

8. Shirasaki H, Nishikawa M, Adcock IM, et al. Expression of platelet-activating factor receptor mRNA in human and guinea pig lung. Am J Respir Cell Mol Biol 1994:10:533-7.

9. Sumitomo T, Nakata M, Yamaguchi M, et al. S-carboxymethylcysteine inhibits adherence of Streptococcus pneumoniae to human alveolar epithelial cells. J Med Microbiol 2012:61:101-8. 